\title{
Catalogue Summary
}

(The numbers relate to the Catalogue entries)

1538, Heinrich Vogtherr (Strasbourg), single sheet: female figure: 1

1538, Jost de Negker (Augsburg), single sheet: female figure: 2

1539, Heinrich Vogtherr (Strasbourg), two sheets: male and female figures: 3

1539, Heinrich Vogtherr (Strasbourg), two sheets: male and female figures: 4

[1539], Hans Guldenmundt (Nuremberg), two sheets: male and female figures: 5

1539, Jean Ruelle (Paris), two sheets: male and female figures: 6

1539, Gianantonio dei Nicolini da Sabbio (Venice), two sheets: male and female figures: 7

[1539], Cornelis Bos (Antwerp), two sheets: male and female figures: 8

[1539-40], Cornelis Bos (Antwerp), two sheets: male and female figures: 9

1540, Joannes Crinitus and Cornelis Bos (Antwerp), two sheets: male and female

figures: 10

[1540], Jean Ruelle (Paris), single sheet: female figure: 11

[1540], Jean Ruelle (Paris), two sheets: male and female figures: 12

[c. 1540], [Heinrich Vogtherr or Jacob Frölich (Strasbourg)], single sheet: male figure: 13

[c. 1540], [Heinrich Vogtherr or Jacob Frölich (Strasbourg)], single sheet: male figure: 14

[c. 1540], [Thomas Raynalde (London)], single sheet: female figure: 15

[1540-50], Sylvester van Parijs (Antwerp), two sheets: male and female figures: 16

[1540-50], Sylvester van Parijs (Antwerp), two sheets: male and female figures: 17

[1540-50], Sylvester van Parijs (Antwerp), two sheets: male and female figures: 18

[1540-50], Sylvester van Parijs (Antwerp), two sheets: male and female figures: 19

1544, Jacob Frölich, alias Jacobus Jucundus (Strasbourg), two sheets: male and female

figures: 20

[c. 1545, (London)], single sheet: male figure: 21

1550, Hans Weygel (Nuremberg), single sheet: male figure: 22

1551-1552, Jacob Frölich alias Jacobus Jucundus (Strasbourg), two sheets: male and female figures: 23

1556, Hans Weygel (Nuremberg), two sheets: male and female figures: 24

[c.1559], [Gilles Godet and/or Thomas Geminus] (London), two sheets: one sheet with male and female figures, one sheet with texts ( 3 printed pages, one blank): 25

[1560], Alain de Matonnière (or Mathonière) (Paris), two sheets: male and female figures: 26

[c.1560-65], Michael Peterle (Prague), two sheets: male and female figures: 27

[1562-63], [Gilles Godet], single sheet with male and female figures: 28

1564, Hans Weygel (Nuremberg), two sheets: male and female figures: 29

1573, (Wittenberg), three sheets: skeleton, male figure and female figure: 30

1584, Matthes Rauch (Nuremberg), two sheets: male and female figures: 31 


\section{Catalogue Summary}

1585, Jean de Gourmont (Paris), single sheet with male and female figures: 32

1585, Georg Lang (Nuremberg), single sheet: female figure: 33

[before 1586], Bartholomäus Schönborn (Wittenberg), two sheets: skeleton and male figure: 34

[before 1586], Bartholomäus Schönborn (Wittenberg), three sheets: male figure, female figure and skeleton: 35

[before 1586], Bartholomäus Schönborn (Wittenberg), single sheet: male figure: 36

[1586?, (Wittenberg)], single sheet: male figure: 37

1587, (Venice), two sheets: male and female figures: 38

1588, Georg Lang (Nuremberg), two sheets: male and female figures: 39

1589, Simon Gronenberg (Wittenberg), single sheet: female figure: 40

[1590-1620], Konrad Corthois (Frankfurt), two sheets: male and female figures: 41

1594, Georg Lang (Nuremberg), single sheet: male figure: 42

1599, (London), single leaf: text: 43

1601, Simon Gronenberg (Wittenberg), single sheet: female figure: 44

1605, Georg Lang (Nuremberg), two sheets: male and female figures: 45

1611, Sebastiano Combi (Venice), single sheet: female figure: 46

1613, Stephan Michelspacher [(Augsburg)], three sheets: male and female figures, male figure, female figure: 47

1613, Stephan Michelspacher [(Augsburg)], three sheets: male and female figures, male figure, female figure: 48

1613, Michel de Matonnière (or Mathonière) (Paris), single sheet with male and female figures: 49

1625, Johann Gormann (Wittenberg), single sheet: female figure: 50

1638, Giovanni Battista Combi (Venice), single sheet: female figure: 51

1656, Thomas Warren (London), probably two sheets: one sheet with male and female figures, one sheet with texts ( 3 printed pages, one blank): 52

1658, Peter Stent (London), two sheets: one sheet with male and female figures, one sheet with texts ( 3 printed pages, one blank): 53

1663, Federico Agnelli (Milan), single sheet: male figure: 54

1663, Filippo Ghisolfi (Milan), single sheet: male figure: 55

1666, Antonio Malatesta (Milan), single sheet: female figure: 56

[c.1680-90], probably of German (or Flemish) origin, four sheets (known as The Four Seasons of Humanity): 57

1687, Lorenzo Ferrari (Cremona), single sheet: male figure: 58

n.d., Mathurin Biesmon (Paris), two sheets: one sheet with male and female figures, one sheet with texts ( 3 printed pages, one blank): 59

n.d., Georg Lang (Nuremberg), single sheet: female figure: 60

Probably second half of the sixteenth century, drawing after Vogtherr's female figure (1538): 61

Second half of the sixteenth century, drawing after Hans Guldenmundt (1539), two sheets: male and female figures: 62 